\title{
EL PARADIGMA INCOMPLETO DE E. COSERIU: TAREA PENDIENTE PARA LA TERCERA GENERACIÓN
}

\author{
JÖRN ALBRECHT \\ Universidad de Heidelberg
}

La mayoría de los sabios tiene sus peculiaridades. Dentro de las particularidades de Eugenio Coseriu se cuenta una extrema reticencia para publicar sus trabajos. Aun cuando su obra publicada es más que considerable ${ }^{1}$, es probable que sus manuscritos inéditos la sobrepasen largamente. ${ }^{2}$ Como discípulo de Coseriu de la primera generación (me refiero con ésta sólo a los discípulos alemanes, puesto que la verdadera primera generación comprende, en primer lugar, a los colegas latinoamericanos, entre cuyos representantes se nombrará aquí sólo a Nelson Cartagena) ${ }^{3}$ y editor de dos apuntes de sus cursos ${ }^{4}$, dispongo de una amplia perspectiva sobre toda una serie de proyectos de investigación que mi maestro perfiló, pero que con su inesperada e incluso temprana muerte a los 81 años, no pudo terminar de pulir.

Emprender ese trabajo pendiente es tarea de la tercera generación de los discípulos de Coseriu. En pocas páginas, intentaré esbozar algunas de las tareas más importantes. Para esto me apoyo en mi apreciación sobre la obra de Coseriu, aparecida hace 15 años. Se encuentra en un homenaje de tres volúmenes que publiqué junto con mis colegas Jens Lüdtke y Harald Thun 5 .

\section{LA HISTORIA DE LOS DISCURSOS Y DEL SABER EXPRESIVO}

Con su artículo «Determinación y entorno», Coseriu entregó una de las aportaciones más tempranas a una Lingüística del Texto ante litteram $^{6}$. La moderna lingüística del texto

\footnotetext{
${ }^{1}$ La bibliografía más completa de sus escritos se encuentra en Murguía 2002.

2 Cf. Kabatek 2002

${ }^{3}$ Cf. junto a los numerosos trabajos de Cartagena su tesis doctoral, Cartagena 1972, que fue dirigida y prologada por Coseriu.

${ }^{4}$ Cf. Coseriu ${ }^{3} 1994 ; 2003$

${ }^{5}$ Cf. Albrecht 1988

${ }^{6}$ «Determinación y entorno. Dos problemas de una lingüística del hablar», Romanistisches Jahrbuch VII, 1955/56, 29-54; reimpreso en E. Coseriu: Teoría del lenguaje y lingüística general, Madrid², 1967: 282323.
} 
ha seguido en parte otro camino que el que Coseriu había señalado, tal vez más fácil, pero no precisamente el más rápido para llegar a su meta. Para evitar equívocos, el contexto teóricoconceptual, dentro del cual aparecen los discursos y el saber expresivo, tiene por lo menos que diseñarse. Con respecto al saber lingüístico, Coseriu distingue primeramente entre plano biológico y plano cultural. Por el primero se entiende nada más que la técnica psicofísica necesaria para la producción del lenguaje. Carece de interés, por lo tanto, desde el punto de vista teórico-conceptual. El plano cultural comprende el conocimiento técnico del lenguaje en cuanto "sistema de signos», de sus modalidades universales y de determinadas modalidades históricas, asi como de las posibilidades que ofrece para diversas circunstancias y diversos momentos expresivos. Como se desprende de esta explicación, Coseriu distingue dentro del plano cultural tres escalones o grados del saber lingüístico: universal, histórico, expresivo. Al escalón universal corresponde el saber cómo se habla en general. Tampoco interesa desde el punto de vista teórico-conceptual. El plano histórico corresponde al saber idiomático, es decir, al «saber» hablar un idioma en sentido estricto. Desde la perspectiva histórica es este saber idiomático el objeto de la historia de la lengua. Esta debe diferenciarse claramente de la historia de los discursos, de la cual aquí se trata. En los discursos no se trata de hablar en general, tampoco de hablar una lengua, sino de hablar en una circunstancia, es decir, hablar sobre algo, en un determinado momento práctico, con alguien y en un determinado ambiente. Es éste, precisamente, el escalón particular o circunstancial del lenguaje, al cual corresponde también un tipo especial de saber lingüistico: es el saber hablar de acuerdo con tal o cual circunstancia, el saber estructurar discursos («textos») conformes a las circunstancias. Coseriu mismo subraya que «a falta de mejor término» llama saber expresivo ${ }^{7}$ a este escalón del saber. En otra circunstancia, Coseriu señala que, si bien es cierto, disponemos de muchos datos en el campo de la historia de las lenguas, disponemos de muy pocos en el ámbito de la historia de los discursos. Aquí yace la primera de las tareas por cumplir: la investigación en el ámbito de las tradiciones del discurso. Algunos trabajos previos, aunque seguramente no siempre en el sentido propuesto por Coseriu, ya se han escrito ${ }^{8}$. La dificultad principal de la historia de los discursos consiste, sin duda, en el deslinde de esta disciplina de la historia de la lengua en sentido tradicional. Algunas consideraciones previas, en todo caso en forma muy abstracta, ha emprendido el autor de este artículo en el citado trabajo (cf. nota 8).

\section{UNA «LINGÜÍSTICA ESQUEUOLÓGICA» QUE ESTUDIE EN SU TOTALIDAD LA CONTRIBUCIÓN DEL «CONOCIMIENTO DE LAS COSAS A LA CONFIGURACIÓN Y AL FUNCIONAMIENTO DEL LENGUAJE ${ }^{9}$}

¿Son sintagmas como eine Frau mit Beinen, eine Stadt mit Häusern, ein Wald mit Bäumen, ein Fluß mit Wasser lingüísticamente anormales? Complementos explicativos tales

\footnotetext{
${ }^{7}$ Cito aquí, en parte textualmente, de un voluminoso manuscrito español con el título «La corrección idiomática». Parte de él se encuentra publicado y traducido al alemán en Coseriu 1988 a, 327-364. Cf. igualmente Coseriu ${ }^{3} 1994$, 54-58.

${ }^{8}$ Cf. entre otros Schlieben-Lange/ Weydt 1979; Aschenberg /Wilhelm 2003. Este tomo contiene también un artículo, correspondiente al tema, del autor de estas páginas.

${ }^{9}$ Cf. Coseriu 1981, 11.
}

Odisea, $n^{\circ} 3,2003$ 
como eine Frau mit langen Beinen, eine Stadt mit alten Häusern, ein Wald mit riesigen Bäumen, ein Fluß mit trübem Wasser no lo son seguramente. Los al comienzo llamados sintagmas, se pueden traducir en la mayoría de los idiomas y también —al menos dentro de nuestro ámbito cultural - se consideran anormales. Se trata pues, difícilmente, de un fenómeno de una lengua particular. El hecho de que todos los sintagmas sean aceptables, cuando se les agrega un complemento, se debe al plano universal, al saber cómo se habla en general. En este contexto, en todo caso, bajo un aspecto muy específico: en nuestro habitual hablar sobre el mundo en una determinada lengua, se introduce siempre una parte del conocimiento de las cosas que consideramos como universal y que presumimos existente en nuestros interlocutores. Que una mujer tenga piernas, una ciudad casas, un bosque árboles y un río agua, es tan evidente que en cualquier lengua de nuestro círculo cultural, en la que las mencionadas «cosas» son conocidas, resulta extraño cuando se mencionan expresamente dichos criterios definitorios. Se interpreta como una clase de tautología, que se resuelve de inmediato cuando se especifican a la vez esos rasgos definitorios. Este era sólo un ejemplo muy simple, pero al que recurre frecuentemente Coseriu para explicar lo que él llama lingüística esqueuológica (del griego skéuos, «cosa»). Hace falta un examen sistemático de la manera de cómo nuestro saber general de las «cosas» pasa a la técnica de nuestro hablar ${ }^{10}$. Un muy modesto y elemental antecedente de esta lingüística esqueuológica se presenta en el movimiento «Wörter und Sachen» que investiga a comienzos del siglo XX, especialmente en el ámbito del hablante alemán, las relaciones entre artefactos y dialectos ${ }^{11}$. Asimismo, se podría ver hoy en una parte de la llamada lingüística «cognitiva», una realización de la tarea propuesta por Coseriu, aunque de ningún modo en el mismo sentido. Me refiero aquí, ante todo, a la semántica de estereotipos, que tiene sus raíces en el tercer libro de John Locke An Essay Concerning Human Understanding ${ }^{12}$. Esta lingüística esqueuológica presenta una extraordinaria y pretenciosa tarea, que puede ser realizada sólo por muchos en común trabajo. Sería así, labor de los discípulos de la segunda generación de Coseriu, proponer a los suyos, o sea, a los de la tercera generación, temas relativos a esta materia, en forma de trabajos de tesis y doctorados. ${ }^{13}$

\section{UNA GRAMÁTICA METALINGÜÍSTICA O UNA GRAMÁTICA DE LA HISTÓRICAMENTE TRANSMITIDA TÉCNICA DEL HABLAR SOBRE EL LENGUAJE}

La diferencia entre «lenguaje primario»y «metalenguaje» es antiquísima. Baste recordar aquí las extraordinarias y sutiles ideas con las que San Agustín en su diálogo De magistro aportó al tema o la teoría, probablemente aún más sutil, de la suposición de los escolásticos ${ }^{14}$. Entretanto se han realizado numerosas contribuciones a este tema, en el marco de la lógica y de la teoría del lenguaje. En el interior del «paradigma incompleto» de

\footnotetext{
${ }^{10}$ Cf. entre otros Coseriu 1988c, 98.

${ }^{11}$ Cf. entre otros Vidos 1975, 80-91.

12 Cf. Locke 1975 (1690), especialmente III, 4ss: Putnam 1975; Coseriu 2003, cap. 12.2.

${ }^{13}$ Rolf Kailuweit, un alumno de Jens Lüdtke y con ello, según se le considere, un discípulo de la «segunda» o «tercera» generación, apunta en su aún no publicado trabajo de habilitación, que ya existen apéndices para tal lingüística esqueuológica. Cf. Kailuweit (en prensa), cap. 1 y 3, nota 22.

${ }^{14}$ Cf. Coseriu 2003, cap. 8.2.2; 9.1.5 y 9.3.
} 
Coseriu, se trata, no obstante, de otra cosa. Para formularlo conscientemente de forma paradójica, se trata de un acceso lingüístico primario al fenómeno del metalenguaje.

Para aclarar esto, se tiene que plantear primero una importante diferencia. Coseriu señala con argumentos convincentes que, al igual que en el lenguaje primario, hay que distinguir también en el metalenguaje entre lengua y habla, entre el metalenguaje de la lengua y el metalenguaje del discurso.

La lengua pertenece a la realidad en todas sus formas de aparición, con lo cual ella puede ser objeto del hablar, como todos los restantes objetos y circunstancias. Por esto, en todas las lenguas existen vocablos con significados «relativos a lo lingüístico», o sea, términos como palabra, lengua, idioma, frase etc. Los significados de estas palabras se refieren a lo lingüístico. Son constantes y estables y no dependen de un determinado modo de utilización (suppositio). En tales casos tenemos que ver con el «metalenguaje de la lengua».

Por otra parte, todas las demás palabras — es decir, palabras sin significados relativos al lenguaje - pueden, en principio, utilizarse de tal modo que ellas no se refieran a lo que normalmente significan, sino sólo a sí mismas. Damos aquí un ejemplo muy simple (con mayor rigor se pueden registrar ejemplos mucho más complicados $)^{15}$ : árbol es una palabra española. En tal caso, tenemos que ver con el metalenguaje del discurso, no se trata del árbol como tal entidad, sino del término árbol ${ }^{16}$.

Tanto en el ámbito del metalenguaje de la lengua como también en el del metalenguaje del discurso, hay peculiaridades que hasta ahora han sido descritas sólo esporádicamente $\mathrm{y} \sin$ método.

De este modo, habría que someter en primer lugar los lexemas de la lengua de partida con significado inherentemente metalingüístico a un análisis comparativo: ¿existe en todos los idiomas un vocablo para «Wort»? ¿qué diferencias significativas pueden comprobarse en los lexemas de la lengua primaria (o sea, no influenciados por la terminología) como Wort, word, mot, palabra, parola, vocabulum, verbum etc.? ¿no está el lenguaje técnico de la lingüística posiblemente influido por sutiles diferencias idiomáticas particulares en el ámbito del «metalenguaje de la lengua»?

Pero también en el ámbito del «metalenguaje del discurso» hay muchas peculiaridades hasta ahora insuficientemente investigadas. Como el mismo Coseriu dice:

Denn auch die Metasprache hat spezifische Normen, denen sie unterliegt. So waren z.B.im Altgriechischen alle Wörter der Metasprache Neutra, gleichgültig, welches Genus sie in der Primärsprache hatten: Auch ein Adverb beispielsweise wurde in der Metasprache als sächliches Substantiv behandelt. Und im Spanischen gibt es eine Norm, die besagt, daß alle Wörter der Metasprache artikellos gebraucht werden, wenn von den Wörtern als solchen die Rede ist. ${ }^{17}$

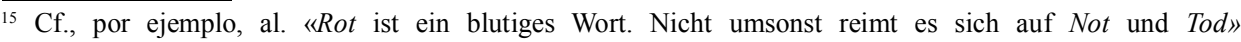
(literalmente en esp. Rot es una palabra sangrienta. No en vano rima con Not y Tod). Relaciones metalingüísticas de este tipo no se dejan traducir en otra lengua, sino simplemente explicar, así, por ejemplo, en español rojo (al. rot) no rima ni con necesidad (al. Not) ni con muerte (al. Tod).

${ }^{16}$ Cf. Coseriu 2003, 130 s.

17 Cf. Coseriu 1988b, 105.
}

Odisea, $n^{\circ} 3,2003$ 
Se pueden señalar otras particularidades de este tipo, aún antes de hacer cualquier examen exhaustivo. Me limitaré aquí solamente a unas pocas. En latín, en el llamado genitivus definitivus: vox libertatis, se encuentra una palabra usada metalingüísticamente. En las lenguas románicas, especialmente en francés, corresponden tales construcciones a las construcciones seguidas por la preposición $\mathrm{DE}$, aunque tal preposición no es obligatoria: le mot de liberté o le mot liberté. Con mucho más frecuencia aparecen tales construcciones, no sólo en las lenguas románicas, sino también en la lengua alemana cuando el signo no se refiere a todo, sino sólo al contenido: la notion de liberté, la noción de libertad, der Begriff der Freiheit.

Sería una tarea muy productiva, investigar las diferencias y semejanzas en ambos campos, en el del «metalenguaje de la lengua»y en el del «metalenguaje del discurso», que abarque en lo posible muchas lenguas.

\section{UNA HISTORIA SUPRAIDIOMÁTICA DEL DISCURSO REPETIDO}

El término discurso repetido fue acuñado por Coseriu primeramente en forma francesa (discours répété $)^{18}$. Constituye parte de las distinciones previas, que deben hacerse para aislar a la lengua funcional, verdadero objeto de la descripción lingüística estructuralista, de la profusión de manifestaciones lingüísticas:

En el estado de lengua «sincrónico» es necesario distinguir dos tipos de tradiciones: la técnica libre del discurso y el discurso repetido. La técnica libre abarca los elementos constitutivos de la lengua y las reglas «actuales» relativas a su modificación y combinación, o sea, las «palabras» y los instrumentos y procedimientos léxicos y gramaticales; el discurso repetido, en cambio, abarca todo aquello que, en el hablar de una comunidad, se repite en forma más o menos idéntica, como discurso ya hecho o combinación más o menos fija, como fragmento por largo o breve que sea, de "ya dicho» ${ }^{19}$.

La distinción discurso repetido de Coseriu no encuentra ninguna correspondencia exacta en el instrumentario conceptual de otras escuelas lingüísticas, porque él une fenómenos que ahí se examinan diferenciada y separadamente: citas, repetición de trozos de textos conocidos como tales, alusiones a textos más o menos conocidos (es decir, la parte substancial de aquello que hoy se entiende normalmente como «intertextualidad»), proverbios, locuciones fijas, fórmulas tradicionales de parangón (por lo tanto, una gran parte de lo que se entiende por «fraseología») $)^{20}$

Criterio definitorio para el discurso repetido es finalmente la capacidad reproductora, es decir, el hecho de que un fragmento de un texto ya terminado se funde en un texto libremente producido. Aunque los discípulos de Coseriu han hecho aportaciones considerables al tema del discurso repetido ${ }^{21}$, pero faltan trabajos para una historia comparada de este fenómeno, en lo posible, en muchos idiomas. En ellos habría que investigar una serie de

\footnotetext{
${ }^{18}$ Cf. Coseriu 1966, 194 ss.

${ }^{19}$ Cf. Coseriu 1981, 297s.

${ }^{20}$ Cf. ibidem 298.

${ }^{21}$ Piénsese por ej. en Thun 1978.
}

Odisea, $n^{\circ} 3,2003$ 
cuestiones. ¿En que caminos desemboca el discurso repetido en la técnica libre del discurso? ¿hay, respecto a eso, diferencias características entre la época de lo oral y de lo escrito? ¿cuál es el porcentaje del discurso repetido en la técnica libre promedio del discurso de diversas lenguas? ¿utiliza el hablante inglés medio más idioms que el francés locutions? ¿aumenta o disminuye la proporción del discurso repetido en la técnica libre media del discurso? ¿qué significa para el hablante, disponer de más o de menos discurso repetido, saberlo más o menos de memoria?. En suma, se trata del papel que el discurso repetido juega en medio del saber idiomático (cf. 1):

El discurso repetido [...] debería estudiarse como una amplia sección autónoma del «saber idiomático».22

\section{LA FUNDAMENTACIÓN DE UNA ESTILÍSTICA NO-IDIOMÁTICA, GENERAL E HISTÓRICA}

El saber expresivo fue estudiado tempranamente dentro del marco de la retórica. Teóricamente se trata en ella de un conocimiento supraidiomático, aunque de hecho se estudió en la antigüedad en uno o, en el mejor de los casos, en dos idiomas, en el griego y el latín:

Después del derrumbe de la retórica como disciplina autónoma [...] varios aspectos del saber expresivo han pasado a ser objeto de la «estilística de la lengua», que a pesar de su nombre, no se limita a lo idiomático, sino que estudia también hechos expresivos (aunque sin distinguirlos de los idiomáticos $)^{23}$.

Típicos para este tipo de estilística que, en el sentido de Coseriu, no separa entre «lengua» y «discurso» o «tradición del discurso», son los trabajos de Leo Spitzer, que han sido ya investigados en el marco del «paradigma» de Coseriu ${ }^{24}$.

Este tipo de estilística, según Coseriu, debe ampliarse y transformarse consecuentemente en una estilística no ligada a una lengua determinada.

Pero todo esto es muy insuficiente, sobre todo desde el punto de vista teórico y en relación con las distinciones que hemos establecido más arriba. En rigor, para el estudio adecuado de los aspectos universales del saber expresivo, se necesitaría una gramática general de los modos circunstanciales de hablar; y para sus aspectos históricos, una disciplina que los estudie y describa como modos expresivos no-idiomáticos, no ligados a una lengua determinada. Es decir que se necesitaría, por un lado, una lingüística de lo circunstancial o, si se quiere, algo como una estilística no-idiomática, general e histórica. Por otro lado, para el estudio de los discursos, se necesitaría una lingüística del texto (o «de los textos»). Mejor dicho, la «lingüística del texto», ya existente como estilística llamada «del habla», debería ser ampliada, de manera que pueda abarcar también el estudio de los modos universales e históricos de los discursos ${ }^{25}$.

Un diseño más o menos detallado de esta empresa ha sido expuesto por Coseriu en sus lecciones sobre lingüística del texto ${ }^{26}$.

\footnotetext{
${ }^{22}$ Cf. Coseriu 1981, 302.

${ }^{23}$ Cf. Coseriu 1988a, 342.

${ }^{24}$ Cf. Aschenberg 1984.

${ }^{25}$ Cf. Coseriu 1988a, 342s.

${ }^{26}$ Cf. Coseriu ${ }^{3} 1994$, especialmente cap.2.5.
}

Odisea, $n^{\circ} 3,2003$ 


\section{LA ELABORACIÓN DE MATERIALES PARA LA DESCRIPCIÓN DE LA NORMA LINGÜÍSTICA}

El concepto de norma (no en el sentido de norma estática o prescriptiva, como se interpreta en la sociolingüística) pertenece a uno de los más importantes resultados del pensamiento lingüístico de Eugenio Coseriu. Su famoso artículo «Sistema, norma y habla» apareció en 1952 en Montevideo. En la extensa lista de sus publicaciones figura entre las primeras, en el octavo lugar. Un vago pre-entendimiento del concepto está ampliamente difundido; bajo una mirada más profunda se comprueba en todo caso, que el concepto no se ha entendido exactamente. Coseriu lo ha desarrollado para corregir, en cierto modo, la conocidísima dicotomía saussureana langue vs. parole. Como se sabe, Saussure creyó poder cubrir con esa dicotomía dos dicotomías parciales. Lo esencial de lo accesorio, por una parte; lo social de lo individual por otra ${ }^{27}$. Coseriu señala en el citado artículo, que ambas dicotomías no son igualmente coincidentes. Lo esencial no coincide necesariamente con lo social, ni tampoco lo accesorio con lo individual ${ }^{28}$. En la norma se trata de lo que en la lengua es común y obligatorio para todos los hablantes, independientemente de si es igualmente esencial o no para su funcionamiento. Debido a ello, la relación entre sistema y norma, desde el punto de vista de su extensión, no se presenta tampoco de modo simple. Si se parte de la relevancia funcional, o sea, de la disyuntiva ¿esencial o accesorio? como criterio superior, es la norma entonces parte del sistema, porque de las posibilidades virtuales puestas en el sistema se realiza históricamente sólo una parte. Así, por ejemplo, se habla en español de un viejo desdentado, pero no de un cuarto desillado, cosa que sería muy legítima según las posibilidades del sistema, si uno alude con ello a un cuarto al que se le han quitado las sillas. Visto desde la perspectiva de lo social obligatorio, es, por el contrario, el sistema una parte de la norma, ya que sólo una parte de lo normal lingüístico es absolutamente necesario para el libre funcionamiento de la lengua. Así, en español se entiende perfectamente a un extranjero, cuando éste en vez de la vibrante $R$ pronuncia una $R$ uvular o velar. Conceptualmente, las lenguas han sido descritas - también en la época pre-estructuralista - atendiendo ante todo a la relevancia funcional, muy poco en cuanto a lo usual. Sería una tarea muy seductora comparar diferentes idiomas atendiendo a la relación de lo funcional relevante y de lo supraindividual normal. Un idioma tan normativo como el francés clásico prescribe una gran cantidad de fenómenos que son totalmente irrelevantes para el funcionamiento del sistema. En alemán, el predominio de la norma, en el sentido de Coseriu, es más débil. En muchos casos, el alemán construye espontáneamente palabras compuestas, sin preocuparse demasiado, si estas construcciones se encuentran o no en un diccionario. En francés ocurre eso muy pocas veces. La frase «Ce n'est pas dans le dictionnaire» es un juicio condenatorio. Tarea de la tercera generación de la escuela de Coseriu sería la de examinar estos fenómenos, que son incluso más complejos de lo que yo aquí, en forma relativamente superficial, he presentado — en el ámbito de la norma de diversas lenguas.

\footnotetext{
${ }^{27}$ Cf. CLG, 30.

${ }^{28}$ Mucho más exacto lo he expuesto en otro lugar: Albrecht ${ }^{22} 2000,32$ y ss.
} 
7. LA FUNDAMENTACIÓN DE UNA VERDADERA, JUSTA, LINGÜÍSTICA CONTRASTIVA CON EL FIN DE OBTENER GRAMÁTICAS Y DICCIONARIOS PARA LA TRADUCCIÓN

La gramática contrastiva, que representó en los años 60 y 70 del siglo pasado a uno de los campos de trabajo más fructíferos dentro de la filología, estuvo al servicio de la didáctica de lenguas extranjeras. Esa forma de gramática comparativa se basaba en una simple idea. La intensidad, que se emplea en la transmisión de determinadas estructuras del idioma que se aprende, depende en gran medida del idioma materno del que la estudia. Así, por ejemplo, es muy positivo transmitir cabalmente las más importantes reglas de concordancia del español a los estudiantes alemanes del español. Para los franceses que aprenden español, basta en cambio, señalar el hecho de que no hay concordancia entre el antepuesto objeto acusativo y el participio en frases del tipo la robe que j'ai mise. Las restantes reglas de concordancia son más o menos iguales en ambas lenguas y los estudiantes franceses del español se divertirán, en cierto modo, cuando se les enseñe que el adjetivo usado predicativamente se rige por el sujeto en género y número ${ }^{29}$.

Las gramáticas para la traducción y, hasta cierto grado, también los diccionarios para la traducción que exige Coseriu tienen en principio otra meta que las normales gramáticas contrastivas y los diccionarios bilingües:

In der Übersetzung geht es aber nicht um unmittelbare Entsprechungen zwischen den Bedeutungen verschiedener Sprachen, sondern von Bezeichnungen in einer Sprache im Vergleich mit einer anderen Sprache, und zwar je nach Bezeichnungsäquivalenz, d.h. um Bedeutungsvarianten... ${ }^{30}$

En la gramática para la traducción se trata pues, de elaborar determinadas reglas de proyección, una tarea que siempre ha jugado un cierto papel en la lexicografía bilingüe, aunque no se haya cumplido con la sistemática exigida por Coseriu. Las unidades o configuraciones («taxemas») de la lengua de partida dada deben examinarse sistemáticamente con vista a establecer cuáles configuraciones les corresponden en la lengua meta, tomando como base, lo que es usual en la lexicografía bilingüe desde hace mucho tiempo, contextos típicos ideales. Esto se podría ilustrar mediante un ejemplo del francés y del alemán, que no proviene de Coseriu, sino del autor de estas líneas:

Il tombait comme au fond d'un puits, entre deux parois de terre verticales. [Caía como al fondo de un pozo, entre dos paredes verticales de tierra]

Una traducción de esta frase al alemán podría ser: «Er fiel wie auf den Boden eines Brunnens, zwischen zwei senkrechten Erdwänden»».

\footnotetext{
${ }^{29}$ La gramática, hasta ahora, más voluminosa e incluso, en cierto sentido, la única gramática contrastiva utilizable del español - alemán es la de Cartagena/ Gauger 1989. Ella pertenece en muchas partes al tipo clásico de gramática contrastiva, muestra no obstante, en algunos capítulos bastantes rasgos de una gramática para la traducción del tipo que se bosqueja a continuación.

${ }^{30}$ Coseriu 1988d, 320.
}

Odisea, $n^{\circ} 3,2003$ 
Comparemos ahora la misma frase con otro tiempo verbal:

Il tomba comme au fond d'un puits, entre deux parois de terre verticales. [Cayó al fondo de un pozo, entre dos paredes verticales de tierra]. «Er fiel wie auf den Boden eines Brunnens, zwischen zwei senkrechte Erdwände».

Se corresponden así — prescindiendo totalmente de consideraciones macrocontextualesdos tiempos del francés (y del español) y dos casos del alemán. El aspecto durativo del imparfait se reproduce, en este caso, satisfactoriamente a través del dativo, el aspecto puntual del passé simple a través del acusativo. Una gramática para la traducción del tipo que patrocina Coseriu, debería destacar y ejemplificar sistemáticamente este tipo de correspondencias entre el francés y el alemán.

En principio esta técnica se conoce desde hace mucho tiempo. Un hermoso ejemplo de ello lo presenta la llamada «estilística latina» de Menge, que contiene ejemplos tan elegidos que, después de casi un siglo, se ha podido editar de nuevo:

Solche deutsche Adverbia, welche nicht ein einzelnes Wort des Satzes näher bestimmen, sondern den ganzen Gedanken affizieren und zugleich ein Urteil des Subjekts enthalten [scil. sog. Satzadverbien], werden durch ein entsprechendes Verbum regens ausgedrückt:

Periclem filium fuisse constat nobilissimorum parentum.

«Perikles war anerkanntmaßen der Sohn hochadeliger Eltern».

Apparet vos esse commotos.

"Ihr seid augenscheinlich tief bewegt $»^{31}$.

Un ejemplo modelo, para esta temprana forma de gramática para la traducción, es también la Grammaire espagnole de Jean Bouzet. Pese a que ella es teóricamente ingenua, lo que se muestra, por ejemplo, en aseveraciones tales como: «L'espagnol ne possède pas le verbe devenir et le traduit par des équivalents». Pero a esta comprobación tan simple, siguen ejemplos excelentes que muestran bajo qué condiciones del contexto puede este verbo corresponderse con ponerse, volverse, hacerse, venir a ser, llegar a ser, acabar por $\operatorname{ser}^{32}$.

Coseriu comprueba acertadamente que las gramáticas para la traducción - en la medida en que se trata de problemas puramente léxicos - presentan proyectos de diccionarios para la traducción, que se pueden realizar sin problemas y que no contienen ninguna dificultad insuperable en sentido teórico. ${ }^{33} \mathrm{Su}$ compilación presenta únicamente una extraordinariamente difícil y ambiciosa tarea en la práctica. Aquí se llama de nuevo a los discípulos de la segunda generación para que dividan proporcionalmente esta tarea, de tal modo que se pueda llevar a cabo en forma de trabajos de tesis y doctorados y los entreguen a sus alumnos, o sea, a la tercera generación de Coseriu.

\footnotetext{
${ }^{31}$ Menge ${ }^{7} 1900,325$

${ }^{32}$ Cf. Bouzet 1976, 250 ss.

${ }^{33}$ Cf. Coseriu 1988d, 324.
} 


\section{UN INVENTARIO DE LAS EXPRESIONES QUE EXISTEN EN LOS DIFERENTES IDIOMAS PARA LA VALORACIÓN DE LOS DISCURSOS}

En el muy elaborado manuscrito El problema de la corrección idiomática, citado aquí a menudo, y del cual hasta ahora sólo una parte ha sido publicada y traducida al alemán (cf. nota 7), se habla extensamente de la valoración de lo hablado. Si bien es cierto que Coseriu se interesa aquí en primer término por determinar el lugar de «lo correcto» en la escala de valoración de lo hablado, señala una y otra vez la necesidad de examinar las expresiones existentes en los diversos idiomas para dicha valoración, independientemente de si se remiten o no a la corrección idiomática. Esta tarea propuesta por Coseriu se puede aquí sólo bosquejar.

Por lo pronto, hay que resaltar que se trata exclusivamente de juicios metalingüísticos de primer grado, es decir, de una valoración de lo hablado por el hablante, no por los lingüistas y, por consiguiente, de una valoración de lo hablado que se efectúa según las normas válidas en la comunidad idiomática que los emite.

Hay dos tipos diferentes de valoración:

a) juicios que se refieren a la calidad misma de los discursos y esto en todos los escalones. ${ }^{34}$ Así, se puede hablar en el escalón biológico de una voz armoniosa, musical, sonora o poderosa; en el escalón elocucional, de rigor, claridad, lucidez, de lo conciso, lo brillante etc. del hablar de alguien. En el escalón idiomático se suele hablar de la riqueza o pureza del hablar y a eso se añade el nombre del idioma en cuestión, es decir: habla un español muy rico, muy matizado, muy puro, etc. Juicios de este tipo son a menudo, difíciles de diferenciar de los que tienen su validez en el escalón expresivo, en donde se trata de la particular habilidad de un hablante para referirse a tal o cual tipo de circunstancias: «Este sí que sabe hablar de...»

b) junto a los juicios que aluden a la cualidad del discurso — por falta de lugar se señalan aquí sólo los positivos - hay algunos, en los cuales se valoriza el discurso que representa el ser intelectual y moral del hablante; su educación y su cultura, su estado de ánimo y sus intenciones. Se trata así del discurso como acción, como acto de conducta que, como tal, puede corresponder o no corresponder a determinadas normas y convenciones de conducta social, concordar o no con las costumbres, los intereses, el estado de ánimo del oyente, y provocar en éste diversas reacciones. Se habla entonces de un discurso inteligente o necio, profundo o superficial, denso o hueco, agudo o ingenuo, moral o inmoral, noble o vil, valiente o cobarde, etc. Juicios de este tipo deben examinarse, según Coseriu, en el centro de la semántica estructural de los diversos idiomas. Con mucha frecuencia, estos juicios son aplicables a los discursos y, al mismo tiempo, a los autores de estos discursos. Pero no siempre ocurre esto. Así, en alemán, se puede hablar sin más de una mujer valiente que ha sostenido un valiente discurso; también se puede hablar de un hombre cobarde, pero difícilmente de un cobarde discurso ${ }^{35}$.

\footnotetext{
$\overline{{ }^{34} \text { Cf. arriba, nota } 7 .}$

${ }^{35}$ Cf. Coseriu 1988a, 343-349
}

Odisea, $n^{\circ} 3,2003$ 


\section{CONCLUSIÓN}

He podido aquí transmitir sólo una impresión de las futuras tareas que Coseriu ha propuesto a sus discípulos y que, hasta ahora nadie ha tomado seriamente. Otros grandes complejos de temas han sido trabajados, desde hace tiempo, por los discípulos de diversas generaciones. Así, por ejemplo, el ciclo de sus lecciones, dictadas a lo largo de años, sobre la historia de la lingüística románica ha encontrado su mejor expresión en las manos de Reinhard Meisterfeld ${ }^{36}$. La historia de la filosofía del lenguaje la ha tomado el mismo autor de estas páginas ${ }^{37}$. Nelson Cartagena ha utilizado con gran agudeza la teoría de Coseriu sobre el entorno que fue desarrollada por primera vez en Determinación y entorno, para los objetivos de la teoría de la traducción ${ }^{38}$. Uno de los campos, que a Coseriu mucho le interesaba, la semántica estructural, fue bajo muchos aspectos perfeccionada por su discípulo Horst Geckeler, muerto a muy temprana edad ${ }^{39}$.

Para terminar, permítaseme señalar algunos grandes temas, que caracterizan el pensamiento de Coseriu y en los que igualmente se distancia de las corrientes principales no sólo de la ciencia lingüística y la teoría del lenguaje sino también de la moderna teoría científica en su conjunto.

- Coseriu estuvo fuertemente convencido de que en el ámbito de la cultura humana, del mondo civile en el sentido de Vico, sólo tiene validez la causa finalis aristotélica. Como digno de la ciencia se considera hoy por lo general sólo la causa efficiens, o sea, la causalidad en sentido estrecho. Para Coseriu en cambio solo la causa finalis constituye el ámbito de la cultura, que es para él, al mismo tiempo, ámbito de la libertad (libertad se entiende aquí como ámbito no sujeto a la determinación causal).

La causa final no debe ser confundida, por lo demás, con la teleología en sentido habitual.

-De especial significado es también su tipología lingüística, difícil de entender y, hasta ahora, — según mi opinión — no adecuadamente comprendida. Para Coseriu el tipo lingüístico representa la manifestación abstracta de algunos principios fundamentales de las lenguas. Por consiguiente, aparece en el lugar superior en la escala de tres escalones TipoSistema- Norma. La tipología de Coseriu quiere ser «tipología funcional de lenguas reales». Para él la clásica tipología morfosintáctica del siglo XIX sería pues, sólo una tipología del «procedimiento lingüístico» y por ello sólo una tipología parcial.

-El tema realmente dominante en Coseriu está representado por su distinción — que seguramente se remite a Vico — entre naturaleza y cultura. En su teoría del lenguaje, ella se manifiesta en su profundo convencimiento de que no sólo se puede distinguir entre mundo y lenguaje, sino que se debe hacerlo. Al igual que Ferdinand de Saussure, aunque también de modo menos radical y con mucho más reservas, Coseriu se preocupó durante toda su vida de conceder al lenguaje cierta autonomía. Una distinción entre conocimiento lingüístico y conocimiento enciclopédico, tal como se entiende más o menos dentro de nuestra

\footnotetext{
${ }^{36}$ Cf. Coseriu /Meisterfeld 2003

${ }^{37}$ Cf. Coseriu 2003.

38 Cf. Cartagena 1995.

${ }^{39}$ Representante para muchos otros, señalamos aquí sólo a uno de sus trabajos, de gran significación teórica de sus últimos años : Geckeler 1999.
}

Odisea, $n^{\circ} 3,2003$ 
cultura europea continental y también en la práctica lexicográfica, cuestionada radicalmente, sin embargo, por investigadores ingleses y americanos ${ }^{40}$, fue defendida por Coseriu con tal despreocupación que, más de una vez, puso en apuros incluso a sus propios discípulos. Piénsese aquí en la distinción entre «significado y designación».

—En la actual situación de la lingüística, determinada totalmente por el llamado «viraje cognoscitivo», la distinción entre «mundo» y «lenguaje» adquiere nuevo sentido. Por una parte, el lenguaje se presenta como ergon, como «forma social objetiva», en la que, con métodos de carácter intersubjetivo, se pueden comprobar ciertas características; por otra parte, el lenguaje aparece como dynamis, como potentia y también como energia y como actio, o sea, interpretados con los medios de la psicología, como capacidad lingüística y como actividad lingüística. Con especial agudeza, Coseriu se pronunció, consecuentemente, en contra de la llamada «semántica de prototipos», la que se puede igualmente considerar como ejemplo prototipo para este nuevo comienzo de la lingüística. Coseriu no hizo esto, porque estuviera totalmente en desacuerdo con dicho enfoque, sino porque tenía que rechazar las pretensiones de una corriente que se presentaba como la verdadera semántica léxica, a la que creía reemplazar, en vez de entenderse como disciplina complementaria de ella, desde una perspectiva diferente, lo que Coseriu sin duda alguna habría aceptado ${ }^{41}$.

\section{BIBLIOGRAFÍA}

AlBRECHT, Jörn. 1988. «tà ónta hôs éstin légein: Über die Schwierigkeit, die Dinge zu sagen, wie sie sind, und andere davon zu überzeugen. Zur Einführung in Energeia und Ergon, 1 11». Jörn Albrecht, ed. Energeia und Ergon. Sprachliche Variation Sprachgeschichte Sprachtypologie, Band I: Schriften von Eugenio Coseriu (1965 1987). Tubinga 1988, XVII XLV.

—1997. «FünfThesen zur kognitiven Semantik». Ulrich Hoinkes/Wolf Dietrich, eds. Kaleidoskop der Lexikalischen Semantik. Tubinga: 19-30.

2000. Europäischer Strukturalismus. Ein forschungsgeschichtlicher Überblick, 2. völlig überarbeitete und erweiterte Fassung. Tubinga 2000 (=UTB 1487)

Aschenberg, Heidi. 1984. Idealistische Philologie und Textanalyse. Zur Stilistik Leo Spitzers. Tubinga.

Aschenberg, Heidi/Wilhelm, Raymund. 2003. Romanische Sprachgeschichte und Diskurstraditionen. Tubinga.

Bouzet, Jean. 1976. Grammaire Espagnole. Paris

Cartagena, Nelson. 1972. Sentido y estructura de las construcciones pronominales en español. Concepción.

Cartagena, Nelson/Hans-Martin Gauger. 1989. Vergleichende Grammatik SpanischDeutsch. Teil 1: Phonetik und Phonologie. Nominalflexematik, Verbalflexematik,

\footnotetext{
$\overline{40}$ Cf., por ejemplo, Haiman 1980.

${ }^{41}$ Cf. Albrecht 1997, Coseriu 1987.
}

Odisea, $n^{\circ} 3,2003$ 
Verbalphrase. Teil 2: Nominal und Pronominalphrase, Wortbildung. Zusammenfassung der wichtigsten grammatischen Unterschiede. Vom Inhalt zu den Formen. Falsche Freunde. Mannheim/Viena/Zurich (= Duden Sonderreihe Vergleichende Grammatiken, Bd. 2)

CARTAgena Nelson. 1995. «Die Grundfunktionen der Sprache und die Übersetzung». Realities of Translating. Anglistik \& Englischunterricht. Heidelberg: Universitätsverlag C. Winter: 247-275.

Coseriu, Eugenio. $1967(1955 / 56)^{2} \cdot$ «Determinaciön y entorno. Dos problemas de una lingüística del hablar». Teoria del lenguaje y lingüística general. Cinco estudios. Madrid: 282-323.

1966. «Structure lexicale et enseignement du vocabulaire». Actes du premier colloque international de linguistique appliquée. Nancy (= Annales de l'Est, Mémoire no. 31): 175-217.

_1981. Lecciones de lingüística general. Madrid.

-1987. «Palabras, cosas y términos». In Memoriam Inmaculada Corrales. Santa Cruz de Tenerife: Universidad de la Laguna: 175-185.

-1988'. «Die Ebenen des sprachlichen Wissens. Der Ort des 'Korrekten' in der Bewertungsskala des Gesprochenen». Jörn AlBREcht, ed. Schriften von Eugenio Coseriu (1965 1987). Eingeleitet und herausgegeben von Jörn Albrecht. Tubinga (=Energeia und Ergon, Bd. 1): 327364.

1988b. «Über den Strukturalismus hinaus». Energeia und Ergon I, op. cit., 103-108.

—1988c. «Interdisziplinarität und Sprache». Energeia und Ergon 1, op. cit., 81-101. -1988d. «Kontrastive Linguistik und Übersetzung: Ihr Verhältnis zueinander». Energeia und Ergon I, op. cit., 311-326.

${ }^{3}$ 1994. Textlinguistik. Eine Einführung. Herausgegeben und bearbeitet von Jörn Albrecht. Tubinga.

2003. Geschichte der Sprachphilosophie. Von den Anfängen bis Rousseau. Neu bearbeitet und erweitert von Jörn Albrecht. Mit einer Vor-Bemerkung von Jürgen Trabant. Tubinga.

Coseriu, Eugenio/Reinhard Meisterfeld. 2003. Geschichte der romanischen Sprachwissenschaft. Bd. 1: Von den Anfängen bis 1492. Tubinga.

GeCKeler, Horst. 1998. «Le champ lexical et les parties du discours». Gerd Wotjak, ed. Teoria del campo y semántica lexical/Théorie des champs et semántica lexicale. Fránfort del Meno: 49-63.

Haiman, John. 1980. «Dictionaries and Encyclopedias». Lingua 50 (1980): 329-357.

KABATEK, Johannes. 2002. «Die unveröffentlichten Manuskripte Eugenio Coserius eine Projektskizze». Adolfo Murguia, ed., op. cit., 111-124. 
KAILUwEIT, Rolf. 2003. Linking. Zum Verhältnis von Syntax und Semantik französischer und italienischer Gefühlsverben. Habilitationsschrift Heidelberg (en prensa).

Locke, John. 1975 (1690). An Essay Concerning Human Understanding, edited with a foreword by Peter H. Nidditsch. Oxford: Erstausgabe 1690.

Murguía, Adolfo, ed. 2000. Sprache und Welt. Festgabe für Eugenio Coseriu zum 80. Geburtstag. Tubinga.

Menge, Hermann. 1900. Repetitorium der lateinischen Syntax und Stilistik. Wolfenbüttel.

Putnam, Hilary. 1975. «The Meaning of Meaning». Mind, Language and Reality. Philosophical Papers, vol. 2, Cambridge

SAussure, Ferdinand de. Cours de linguistique générale. Paris, varias ediciones (=CLG).

Schlieben Lange, Brigitte/Harald Weydt. 1979. «Streitgespräch zur Historizität von Sprechakten (mit Beiträgen von Eugenio Coseriu und Hans Ulrich Gumbrecht)». Linguistische Berichte 60: 65-78.

Thun, Harald. 1978. Probleme der Phraseologie. Untersuchungen zur wiederholten Rede mit Beispielen aus dem Französischen, Italienischen, Spanischen und Rumänischen. Tubinga.

Vidos, B. E. 1975. Handbuch der romanischen Sprachwissenschaft. Múnich. 